Research Paper

\title{
Antibacterial efficacy of Nisin, Pediocin 34 and Enterocin FH99 against Listeria monocytogenes and cross resistance of its bacteriocin resistant variants to common food preservatives
}

\author{
G. Kaur, T.P. Singh, R.K. Malik \\ Dairy Microbiology Division, National Dairy Research Institute, Karnal, India.
}

Submitted: March 16, 2011; Approved: July 2, 2012.

\begin{abstract}
Antilisterial efficiency of three bacteriocins, viz, Nisin, Pediocin 34 and Enterocin FH99 was tested individually and in combination against Listeria mononcytogenes ATCC 53135. A greater antibacterial effect was observed when the bacteriocins were combined in pairs, indicating that the use of more than one LAB bacteriocin in combination have a higher antibacterial action than when used individually. Variants of Listeria monocytogenes ATCC 53135 resistant to Nisin, Pediocin 34 and Enterocin FH99 were developed. Bacteriocin cross-resistance of wild type and their corresponding resistant variants were assessed and results showed that resistance to a bacteriocin may extend to other bacteriocins within the same class. Resistance to Pediocin 34 conferred cross resistance to Enterocin FH 99 but not to Nisin. Similarly resistance to Enterocin FH99 conferred cross resistance to Pediocin 34 but not to Nisin. Also, the sensitivity of Nisin, Pediocin 34 and Enterocin FH99 resistant variants of Listeria monocytogenes to low $\mathrm{pH}$, salt, sodium nitrite, and potassium sorbate was assayed in broth and compared to the parental wild-type strain. The Nisin, Pediocin 34 and Enterocin FH99 resistant variants did not have intrinsic resistance to low $\mathrm{pH}$, sodium chloride, potassium sorbate, or sodium nitrite. In no case were the bacteriocin resistant Listeria monocytogenes variants examined were more resistant to inhibitors than the parental strains.
\end{abstract}

Key words: Listeria monocytogenes, Nisin, Pedioin 34, Enterocin FH99, cross resistance.

\section{Introduction}

Bacteriocins are ribosomally-synthesized antimicrobial peptides or proteins, produced mainly by lactic acid bacteria (LAB). Several LAB bacteriocins with broad spectra of inhibitory activity offer potential applications in food biopreservation (Galvez et al., 2008). Several reports have described the emergence of nisin resistant mutants of Listeria monocytogenes on exposure of the nisin-sensitive cells to relatively high nisin concentrations (Ming and Daeschel, 1993, 1995; Davies et al., 1996; Verheul et al., 1997). Resistance has been correlated to an altered fatty acid composition (Ming and Daeschel, 1993; Mazotta and Montville, 1997) and altered phospholipids composition (Ming and Daeschel, 1995; Crandall and Montville, 1998). Studies have revealed the stability of bacteriocin resistance phenomenon (Rekhif et al., 1994; Dykes and Hastings, 1998) and it has been reported to occur at either a low or a high level. In Listeria monocytogenes and Enterococcus faecalis, low-level resistance has been reported to be due to alterations in membrane lipid composition (Vadyvaloo et al., 2002; Naghmouchi et al., 2006). High-level resistance has been attributed to results from the inactivation of the mptACD operon and due to several changes in protein synthesis (Ming and Daeschel, 1993; Gravesen et al., 2000; Ramanath et al., 2000; Dalet et al., 2001; Hechard et al., 2001; Gravesen et al., 2002; Calvez et al., 2007).

The development of bacteriocin resistance might hinder application of bacteriocins in food preservation. The aim of the present study was to show that bacteriocins of lactic acid bacteria in combination have a higher antibacterial action against Listeria than when used individually. Further, it has been shown that resistance to a bacteriocin 
may extend to other bacteriocins within the same class. Also, in this study we examined the sensitivity of bacteriocin resistant variants of Listeria monocytogenes to sodium chloride, low $\mathrm{pH}$, sodium nitrite, and potassium sorbate in comparison with the parental wild-type strains to determine if resistance to nisin confers cross resistance to these common food preservatives.

\section{Materials and Methods}

\section{Bacterial strains and culture conditions}

Enterococcus faecium FH99, bacteriocinogenic strain was a isolate from human faeces (Gupta et al., 2010). Pediococcus pentosaceous 34, a bacteriocinogenic strain was an isolate from cheddar cheese. Pediococcus acidilactici LB 42 (a sensitive strain used for detection of bacteriocin producers), was obtained from Prof. Bibek Ray, Department of Animal Science, University of Wyoming, Laramie Wyoming, USA. L. monocytogenes ATCC 53135 was obtained from American Type Culture Collection (ATCC).

\section{Bacteriocins}

One hundred milliliter aliquots of MRS broth (De Man et al., 1960) (pH 6.5) (HiMedia, Mumbai) were inoculated with active culture of E. faecium FH99 (Gupta et al., $2010)$ and $P$. pentosaceus $34(1 \%)$ and incubated at $37{ }^{\circ} \mathrm{C}$ for $24 \mathrm{~h}$. Cell free culture supernatant (CFCS) were prepared by centrifugation of the cultures in refrigerated centrifuge at $10,000 \mathrm{rpm}$ for $10 \mathrm{~min}$. The supernatant was filter sterilized by passing through a $0.2 \mu \mathrm{m}$ (Millipore), $45 \mathrm{~mm}$ diameter membrane filter and used for partial purification after neutralization. Crude enterocin FH99 and pediocin 34 were precipitated from broth media by $60 \%$ ammonium sulphate precipitation and the precipitates were dissolved in sterilized Milli Q water. Enterocin FH99 and Pediocin 34 were purified by the method earlier described by Gupta et al. (2010). Nisin A (Nisaplin $\left.{ }^{\circledR}\right)$ was obtained from Danisco (Gurgaon, India). Nisin stock solutions were prepared from pure nisin in $0.02 \mathrm{~N} \mathrm{HCl}$ and autoclaved.

\section{Measurement of activity units $\left(A U \mathrm{~mL}^{-1}\right)$}

The antibacterial activity of nisin, pediocin 34 and enterocin FH99 was obtained using the spot on lawn assay as described by Ulhman et al. (1992), against $P$. acidilactici LB 42. Five microlitres of serial dilutions of the partially purified bacteriocin of E. faecium $\mathrm{FH} 99$ and $P$. pentosaceus 34 grown in MRS broth (De Man et al., 1960) were spotted on the Tryptone Glucose Yeast Extract (TGE) agar plates (Biswas et al., 1991) (1.5\% agar). Before spotting, TGE agar plates were overlaid with TGE soft agar $(0.75 \%)$ seeded with actively growing cells of the test organism. Plates were kept undisturbed for 3-4 h for diffusion of bacteriocin through agar and then incubated. The sensitivity of the strain in question was evaluated by checking for clear zones around the spots. Three independent replicates of experiment were done. The activity units of the culture broth were calculated using the following formula and expressed as activity units per $\mathrm{mL}$ :

Activity Units per $\mathrm{mL}\left(\mathrm{AU} \mathrm{mL}^{-1}\right)=200 \times$ Reciprocal of highest dilution that gave a clear zone

\section{Bacteriocin susceptibility test and determination of Minimum Inhibitory Concentrations (MICs)}

The inhibitory spectrum of activity was obtained using the spot on lawn assay as described by Ulhman et al. (1992) against L. monocytogenes ATCC 53135. Five microlitres of the partially purified bacteriocin of $E$. faecium FH 99 and Pediococcus pentosaceus 34 grown in MRS broth (De Man et al., 1960) was spotted on the plates TGE agar plates (Biswas et al., 1991) (1.5\% agar). Before spotting, TGE agar plates were overlaid with TGE soft agar $(0.75 \%)$ seeded with actively growing cells of the test organism. Plates were kept undisturbed for 3-4 h for diffusion of bacteriocin through agar and then incubated. The sensitivity of the strain in question was evaluated by checking for clear zones around the spots. For MIC determinations, $5 \mu \mathrm{L}$ of a 1:2 dilution series of a bacteriocin solution was placed in wells. The Minimum Inhibitory Concentration (MIC) value was interpreted as the lowest concentration of bacteriocin that resulted in a clear inhibition halo after $18 \mathrm{~h}$ incubation at $37^{\circ} \mathrm{C}$. The MIC was defined as the lowest concentration of bacteriocin that induced an inhibition zone.

\section{Kinetics of cell growth inhibition by bacteriocins}

Overnight cultures of L. monocytogenes ATCC 53135 was inoculated into fresh BHI broth tubes $(1 \%)$ containing either nisin, pediocin 34 or enterocin FH99. These bacteriocins were used individually or in combination; the mixture contained the calculated MICs of each bacteriocin. Additionally, the efficacy of nisin, pediocin 34 and enterocin FH99 in combination (half the concentration of MICs for each bacteriocin) was also evaluated. The concentrations of the bacteriocins used when tested against the target organism alone and in different combinations, respectively have been mentioned in footnote of Table 1. At different time intervals ( $1 \mathrm{~h}, 2 \mathrm{~h}, 4 \mathrm{~h}, 6 \mathrm{~h}$ and $24 \mathrm{~h}$ ) the survivors were enumerated on Brain heart infusion (BHI) agar medium after appropriate dilutions in saline, and colonies were counted after $24-48 \mathrm{~h}$ of incubation at $37^{\circ} \mathrm{C}$. Three independent replicates of experiment were done.

\section{Isolation of spontaneous bacteriocin resistant variants}

Spontaneous resistant mutants of L.monocytogenes ATCC 53135 to nisin, pediocin 34 and enterocin FH99 were isolated after sequential exposure to a bacteriocin concentration 10-fold higher the MIC. 


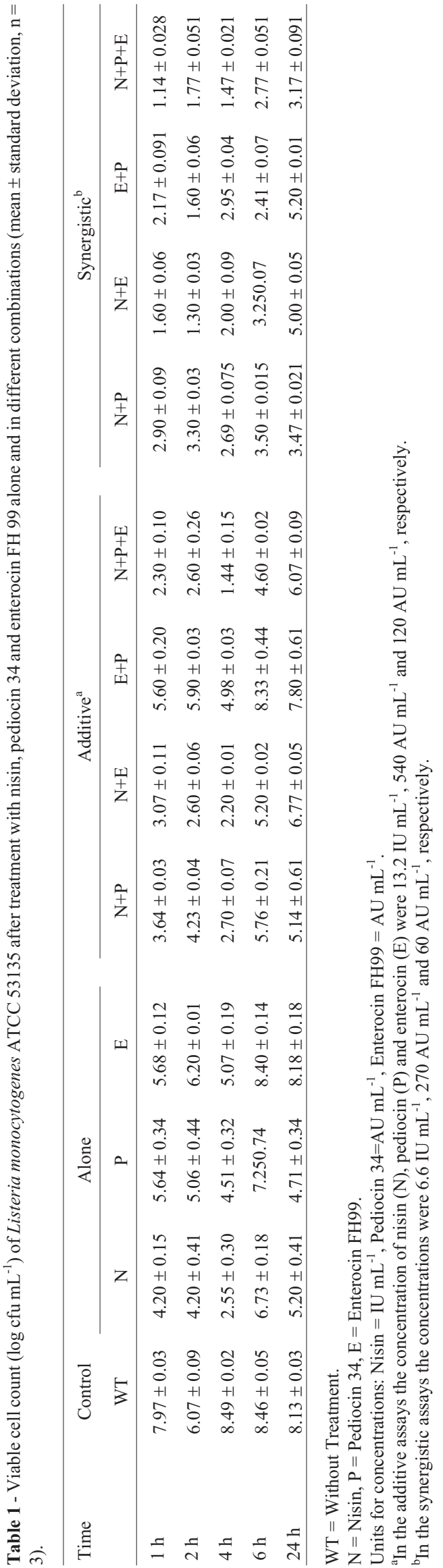

\section{Bacteriocin cross-resistance by Agar diffusion method}

The sensitivity of L. monocytogenes ATCC 53135 and its resistant variants to nisin, pediocin 34 and enterocin FH99 were qualitatively determined by the agar well diffusion method. Briefly, $5 \mathrm{~mL}$ of molten TGE agar containing $0.75 \%(\mathrm{w} / \mathrm{v})$ agar medium were cooled at $47^{\circ} \mathrm{C}$ and seeded with $1 \%(\mathrm{v} / \mathrm{v})$ overnight $\mathrm{BHI}$ culture of L. monocytogenes ATCC 53135 and its nisin, pediocin 34 or enterocin FH99 resistant variants. Seeded agar was then poured onto TGE agar plate and allowed to solidify at room temperature. Wells $(8 \mathrm{~mm})$ were cut in the solidified agar using a sterile metal cork borer and filled with $80 \mu \mathrm{L}$ of sample. The plates were left at $5{ }^{\circ} \mathrm{C}$ for $2 \mathrm{~h}$ to allow diffusion of the tested aliquot and then incubated for $18 \mathrm{~h}$ at $37^{\circ} \mathrm{C}$. Absence or presence of inhibition zones was recorded.

\section{Cross resistance to low $\mathrm{pH}$, potassium sorbate, sodium chloride and sodium nitrite}

Experiments were conducted to examine the sensitivity of bacteriocin resistant variants of $L$. monocytogenes ATCC 53135 to common food preservatives i.e. sodium chloride $(\mathrm{NaCl})$, low $\mathrm{pH}$, sodium nitrite and potassium sorbate in comparison with the parental sensitive-type strains and to determine if resistance to bacteriocins confers cross-resistance to these common food preservatives. BHI broth was supplemented with $\mathrm{NaCl}$ to final concentrations of $1 \%, 2 \%, 8 \%, 9 \%, 10 \%, 11 \%, 12 \%, 14 \%$ and $16 \%$ (wt/vol) (control, $0 \%$ additional $\mathrm{NaCl}$ ); (ii) BHI broth was acidified with concentrated $\mathrm{HCl}$ to final $\mathrm{pH}$ values of 5.2, 5.0, 4.8, 4.6 and 4.4 (control, pH 7.4); BHI broth was supplemented with sodium nitrite to final concentrations of 20 , $25,30,35,40,45,50$ and $55 \mu \mathrm{g} / \mathrm{mL}$ (control, no additional sodium nitrite) and potassium sorbate to final concentrations of 2, 2.5, 3.0, 3.5, 4, 4.5, 5, 5.5 and 6, $\mathrm{mg} / \mathrm{ml}$ (control: no additional potassium sorbate). Three independent replicates of experiment were done.

\section{Results}

\section{Kinetics of cell growth inhibition by bacteriocins}

In the present study, the antibacterial efficacy of nisin, pediocin 34 and enterocin FH99 was evaluated alone as well as in different combinations against $L$. monocytogenes ATCC 53135 in BHI broth. The calculated MICs of the nisin, pediocin 34 and enterocin FH99 against L. monocytogenes were $13.2 \mathrm{IU} \mathrm{mL}^{-1}, 540 \mathrm{AU} \mathrm{mL}^{-1}$ and $120 \mathrm{AU} \mathrm{mL}^{-1}$, respectively. These MICs were used to evaluate the antibacterial effect of bacteriocins alone. In order to evaluate additive and synergistic effect of bacteriocins the different combinations and the concentrations of bacteriocins were used against the target organisms. Nisin was also observed to be most effective in inhibiting the $L$. monocytogenes ATCC 53135, followed by pediocin 34 and 
enterocin FH99. In case of Listeria, it was observed that even when nisin displayed the most rapid inhibitory activity at $1 \mathrm{~h}$, the survivors resumed growth, reaching the highest cell counts at $24 \mathrm{~h}$. The results of the present study indicate that combinations of different bacteriocins produce a more effective antibacterial effect against $L$. monocytogenes ATCC 53135 in comparison to the bacteriocins used alone. When the two non nisin bacteriocins were used together, a higher number of survivors were detected than with the pairs containing nisin. Also, synergistic action was observed between different combinations of bacteriocins when tested against $L$. monocytogenes ATCC 53135. A combination of nisin, pediocin 34 and enterocin FH99 was most effective against $L$. monocytogenes ATCC 53135 (Table 1).

\section{Bacteriocin cross resistance}

The bacteriocin cross resistance profiles of wild type L. monocytogenes and its corresponding nisin, pediocin 34 and enterocin FH99 resistant variants is shown in Table 2. Wild type L. monocytogenes ATCC 53135 showed sensitivity to nisin, pediocin 34 and enterocin FH99. However, pediocin 34 resistant variant showed resistance to enterocin FH99 and not nisin, On the other hand nisin resistant variant retained the sensitivity to pediocin 34 as well as enterocin FH99. Resistance to enterocin FH99 conferred cross resistance to pediocin 34 but not to nisin (Table 2).
Table 2 - Comparison of susceptibility of wild type strains and resistant variants of Listeria monocytogenes ATCC 53135 to nisin, pediocin 34 (P) and enterocin FH99 (E) bacteriocins.

\begin{tabular}{lccc}
\hline Pathogen & Nisin & P & E \\
\hline WT & + & + & + \\
Nr & - & + & + \\
Pr & + & - & - \\
Er & + & - & - \\
\hline
\end{tabular}

WT $=$ wild type, $\mathrm{Nr}=$ nisin resistant, $\mathrm{Pr}=$ Pedioicn 34 resistant, $\mathrm{Er}=$ Enterocin FH99 resistant. No inhibition $=-$, Inhibition $=+$.

\section{Cross resistance of wild type and resistant strains to common food preservatives}

Table 3 and Table 4 shows the viable cell count $\left(\log \mathrm{cfu} \mathrm{mL}^{-1}\right)$ of wild type L. monocytogenes ATCC 53135 and its nisin, pediocin 34 and enterocin FH99 resistant variants at different time intervals after growth in medium with $\mathrm{pH} 4.4,4.8$ and 5.0 and sodium chloride at concentration of $1,2,8,9,10$ and $11 \%(\mathrm{w} / \mathrm{v})$, respectively. Table 5 and Table 6 shows the viable cell count $\left(\log \mathrm{cfu} \mathrm{mL}^{-1}\right)$ of wild type L. monocytogenes ATCC 53135 and its nisin, pediocin 34 and enterocin FH99 resistant variants at different time intervals after growth in medium supplemented with potassium sorbate at concentration of 2.0, 3.0, 4.0, 5.0 and $6.0 \mathrm{mg} / \mathrm{mL}$ and sodium nitrite at concentration of 20 ,

Table 3 - Viable cell count (log cfu $\mathrm{mL}^{-1}$ ) of wild type Listeria monocytogenes ATCC 53135 (WT) and its nisin (Nr) resistant, pediocin 34 resistant and enterocin FH99 resistant variant at different time intervals after growth in medium with $\mathrm{pH} 4.4,4.8$ and 5.0 (mean \pm standard error, $\mathrm{n}=3$ ).

\begin{tabular}{|c|c|c|c|c|c|c|c|}
\hline \multirow[t]{2}{*}{$\mathrm{pH}$} & \multirow[t]{2}{*}{ Culture } & \multicolumn{6}{|c|}{ Time (h) } \\
\hline & & 1 & 2 & 4 & 6 & 8 & 24 \\
\hline \multirow[t]{4}{*}{ Control } & WT & $7.97 \pm 0.03$ & $7.74 \pm 0.03$ & $8.49 \pm 0.13$ & $7.86 \pm 0.03$ & $8.95 \pm 0.02$ & $8.11 \pm 0.09$ \\
\hline & $\mathrm{Nr}$ & $7.95 \pm 0.04$ & $7.71 \pm 0.03$ & $8.47 \pm 0.01$ & $7.83 \pm 0.09$ & $8.93 \pm 0.08$ & $8.04 \pm 0.03$ \\
\hline & $\operatorname{Pr}$ & $7.99 \pm 0.05$ & $7.72 \pm 0.06$ & $8.86 \pm 0.09$ & $7.83 \pm 0.09$ & $8.94 \pm 0.03$ & $8.00 \pm 0.13$ \\
\hline & Er & $7.94 \pm 0.09$ & $7.71 \pm 0.03$ & $8.47 \pm 0.01$ & $7.85 \pm 0.02$ & $8.93 \pm 0.09$ & $8.04 \pm 0.03$ \\
\hline \multirow[t]{4}{*}{4.4} & WT & $3.51 \pm 0.85$ & $4.81 \pm 0.29$ & $3.60 \pm 0.20$ & $3.39 \pm 0.79$ & 2.250 .35 & $3.51 \pm 0.13$ \\
\hline & $\mathrm{Nr}$ & $4.20 \pm 0.04$ & $2.47 \pm 0.12$ & $2.62 \pm 0.32$ & $2.11 \pm 0.81$ & $2.14 \pm 0.61$ & $2.60 \pm 0.20$ \\
\hline & $\operatorname{Pr}$ & $2.11 \pm 0.39$ & $1.60 \pm 0.20$ & $2.27 \pm 0.60$ & $1.77 \pm 0.15$ & $1.11 \pm 0.39$ & $2.00 \pm 0.83$ \\
\hline & Er & $3.34 \pm 0.24$ & $2.34 \pm 0.81$ & $2.46 \pm 0.23$ & $2.32 \pm 0.22$ & $2.90 \pm 0.30$ & $3.17 \pm 0.60$ \\
\hline \multirow[t]{4}{*}{4.8} & WT & $6.17 \pm 0.12$ & $5.73 \pm 0.23$ & $6.04 \pm 0.13$ & $6.68 \pm 0.12$ & $5.88 \pm 0.08$ & $5.90 \pm 0.30$ \\
\hline & $\mathrm{Nr}$ & $4.44 \pm 0.31$ & $4.49 \pm 0.13$ & 3.830 .25 & 4.250 .05 & $3.81 \pm 0.13$ & $4.04 \pm 0.26$ \\
\hline & $\operatorname{Pr}$ & $4.77 \pm 0.51$ & $3.90 \pm 0.30$ & $3.77 \pm 0.15$ & $3.62 \pm 0.23$ & $3.49 \pm 0.13$ & $3.44 \pm 0.31$ \\
\hline & Er & $3.60 \pm 0.20$ & $3.51 \pm 0.13$ & $3.50 \pm 0.51$ & $3.69 \pm 0.04$ & $3.39 \pm 0.79$ & $3.07 \pm 0.91$ \\
\hline \multirow[t]{4}{*}{5.0} & WT & $5.38 \pm 0.61$ & $5.70 \pm 0.01$ & $5.50 \pm 0.14$ & $6.55 \pm 0.30$ & $6.63 \pm 0.34$ & $7.27 \pm 0.60$ \\
\hline & $\mathrm{Nr}$ & $4.81 \pm 0.16$ & $5.00 \pm 0.85$ & $5.41 \pm 0.49$ & 5.250 .27 & 4.770 .25 & $4.00 \pm 0.49$ \\
\hline & $\operatorname{Pr}$ & $4.77 \pm 0.12$ & $4.69 \pm 0.04$ & $4.60 \pm 0.20$ & $4.54 \pm 0.40$ & $4.41 \pm 0.49$ & $4.14 \pm 0.61$ \\
\hline & Er & $4.47 \pm 0.71$ & $4.17 \pm 0.60$ & $3.84 \pm 0.50$ & $4.00 \pm 0.82$ & $3.84 \pm 0.50$ & $3.54 \pm 0.44$ \\
\hline
\end{tabular}

WT $=$ Wild Type, $\mathrm{Nr}=$ Nisin resistant variant, $\mathrm{Pr}=$ Pediocin 34 resistant variant, $\mathrm{Er}=$ Enterocin $\mathrm{FH} 99$ resistant variant. 
Table 4 - Viable cell count (log cfu $\mathrm{mL}^{-1}$ ) of wild type Listeria monocytogenes ATCC 53135 (WT) and its nisin (Nr) resistant, pediocin 34 resistant (Pr) and enterocin FH99 (Er) resistant variant at different time intervals after growth in supplemented with Sodium Chloride at concentration of 1, 2, 8, 9, 10 and $11 \%(\mathrm{w} / \mathrm{v})($ mean \pm standard error, $\mathrm{n}=3)$.

\begin{tabular}{|c|c|c|c|c|c|c|c|}
\hline \multirow{2}{*}{$\begin{array}{l}\text { Concentration } \\
(\% \mathrm{w} / \mathrm{v})\end{array}$} & \multirow[t]{2}{*}{ Culture } & \multicolumn{6}{|c|}{ Time (h) } \\
\hline & & 1 & 2 & 4 & 6 & 8 & 24 \\
\hline \multirow[t]{4}{*}{ Control } & WT & $7.97 \pm 0.03$ & $7.74 \pm 0.03$ & $8.49 \pm 0.13$ & $7.86 \pm 0.03$ & $8.95 \pm 0.02$ & $8.11 \pm 0.09$ \\
\hline & $\mathrm{Nr}$ & $7.95 \pm 0.04$ & $7.71 \pm 0.03$ & $8.47 \pm 0.01$ & $7.83 \pm 0.09$ & $8.93 \pm 0.08$ & $8.04 \pm 0.03$ \\
\hline & $\operatorname{Pr}$ & $7.99 \pm 0.05$ & $7.72 \pm 0.06$ & $8.86 \pm 0.09$ & $7.83 \pm 0.09$ & $8.94 \pm 0.03$ & $8.00 \pm 0.13$ \\
\hline & Er & $7.94 \pm 0.09$ & $7.71 \pm 0.03$ & $8.47 \pm 0.01$ & $7.85 \pm 0.02$ & $8.93 \pm 0.09$ & $8.04 \pm 0.03$ \\
\hline \multirow[t]{4}{*}{1} & WT & $5.34 \pm 0.13$ & $6.07 \pm 0.08$ & $6.30 \pm 0.10$ & $6.78 \pm 0.05$ & $7.22 \pm 0.05$ & $7.17 \pm 0.09$ \\
\hline & $\mathrm{Nr}$ & $4.95 \pm 0.03$ & $4.90 \pm 0.09$ & $5.11 \pm 0.03$ & $7.32 \pm 0.14$ & $6.97 \pm 0.08$ & $6.39 \pm 0.04$ \\
\hline & $\operatorname{Pr}$ & $4.69 \pm 0.07$ & $4.77 \pm 0.06$ & $4.90 \pm 0.09$ & $6.74 \pm 0.08$ & $7.11 \pm 0.06$ & $6.62 \pm 0.09$ \\
\hline & Er & $4.63 \pm 0.08$ & $4.59 \pm 0.15$ & $5.66 \pm 0.08$ & $6.43 \pm 0.04$ & $5.68 \pm 0.07$ & $5.36 \pm 0.17$ \\
\hline \multirow[t]{4}{*}{2} & WT & $5.39 \pm 0.03$ & $6.08 \pm 0.06$ & $6.14 \pm 0.10$ & $6.30 \pm 0.03$ & $7.11 \pm 0.12$ & $6.77 \pm 0.08$ \\
\hline & $\mathrm{Nr}$ & $4.84 \pm 0.08$ & $4.84 \pm 0.09$ & $4.77 \pm 0.08$ & $5.50 \pm 0.05$ & $6.50 \pm 0.10$ & $5.95 \pm 0.09$ \\
\hline & $\operatorname{Pr}$ & $4.68 \pm 0.04$ & $4.39 \pm 0.07$ & $4.98 \pm 0.01$ & $5.96 \pm 0.10$ & $5.97 \pm 0.04$ & $6.30 \pm 0.03$ \\
\hline & Er & $4.46 \pm 0.02$ & $4.65 \pm 0.03$ & $5.82 \pm 0.05$ & $5.50 \pm 0.15$ & $5.44 \pm 0.08$ & $5.00 \pm 0.09$ \\
\hline \multirow[t]{4}{*}{8} & WT & $4.20 \pm 0.12$ & $4.23 \pm 0.09$ & $4.20 \pm 0.13$ & $4.00 \pm 0.06$ & $4.00 \pm 0.19$ & $3.60 \pm 0.06$ \\
\hline & $\mathrm{Nr}$ & $3.69 \pm 0.07$ & $3.60 \pm 0.06$ & $3.30 \pm 0.03$ & $3.60 \pm 0.14$ & $3.07 \pm 0.06$ & $3.04 \pm 0.03$ \\
\hline & $\operatorname{Pr}$ & $3.68 \pm 0.04$ & $3.39 \pm 0.07$ & $3.98 \pm 0.07$ & $3.96 \pm 0.08$ & $3.97 \pm 0.04$ & $3.30 \pm 0.04$ \\
\hline & Er & $3.94 \pm 0.09$ & $3.71 \pm 0.11$ & $3.47 \pm 0.08$ & $3.85 \pm 0.06$ & $3.93 \pm 0.09$ & $3.04 \pm 0.07$ \\
\hline \multirow[t]{4}{*}{9} & WT & $3.95 \pm 0.03$ & $3.47 \pm 0.02$ & $4.00 \pm 0.11$ & $4.69 \pm 0.07$ & $3.60 \pm 0.06$ & $3.69 \pm 0.08$ \\
\hline & $\mathrm{Nr}$ & $3.60 \pm 0.06$ & $3.47 \pm 0.07$ & $3.60 \pm 0.01$ & $3.00 \pm 0.09$ & $3.00 \pm 0.10$ & $3.14 \pm 0.06$ \\
\hline & $\operatorname{Pr}$ & $3.47 \pm 0.07$ & $3.34 \pm 0.03$ & $3.55 \pm 0.06$ & $3.11 \pm 0.04$ & $3.17 \pm 0.09$ & 3.250 .03 \\
\hline & Er & $3.47 \pm 0.11$ & $3.40 \pm 0.06$ & $3.69 \pm 0.08$ & $3.83 \pm 0.10$ & $3.45 \pm 0.02$ & $3.00 \pm 0.10$ \\
\hline \multirow[t]{4}{*}{10} & WT & $3.39 \pm 0.04$ & $3.46 \pm 0.08$ & $3.71 \pm 0.03$ & $3.69 \pm 0.07$ & $3.07 \pm 0.08$ & $3.07 \pm 0.10$ \\
\hline & $\mathrm{Nr}$ & $3.30 \pm 0.03$ & $3.36 \pm 0.11$ & $3.30 \pm 0.06$ & $2.78 \pm 0.05$ & $2.90 \pm 0.09$ & $2.95 \pm 0.03$ \\
\hline & $\operatorname{Pr}$ & $3.27 \pm 0.04$ & 3.250 .03 & $3.20 \pm 0.02$ & $2.95 \pm 0.03$ & $2.99 \pm 0.05$ & $2.07 \pm 0.08$ \\
\hline & Er & $3.14 \pm 0.08$ & $3.17 \pm 0.09$ & $3.49 \pm 0.09$ & $3.36 \pm 0.08$ & $2.95 \pm 0.03$ & $2.81 \pm 0.04$ \\
\hline \multirow[t]{4}{*}{11} & WT & $2.82 \pm 0.05$ & $2.60 \pm 0.06$ & $3.14 \pm 0.08$ & $3.30 \pm 0.06$ & 3.250 .05 & $3.04 \pm 0.09$ \\
\hline & $\mathrm{Nr}$ & $2.69 \pm 0.07$ & $2.47 \pm 0.04$ & $2.47 \pm 0.02$ & $2.50 \pm 0.09$ & $2.77 \pm 0.11$ & $2.84 \pm 0.08$ \\
\hline & $\operatorname{Pr}$ & $2.47 \pm 0.10$ & $2.62 \pm 0.03$ & $2.55 \pm 0.07$ & $2.51 \pm 0.04$ & $2.99 \pm 0.05$ & $2.91 \pm 0.04$ \\
\hline & $\mathrm{Er}$ & $2.97 \pm 0.08$ & $2.54 \pm 0.06$ & $2.49 \pm 0.02$ & $2.86 \pm 0.03$ & $2.95 \pm 0.03$ & $2.79 \pm 0.08$ \\
\hline
\end{tabular}

WT $=$ Wild Type, $\mathrm{Nr}=$ Nisin resistant variant, $\mathrm{Pr}=$ Pediocin 34 resistant variant, Er $=$ Enterocin FH99 resistant variant, ND = Not Detected.

30,40 and $50 \mu \mathrm{g} / \mathrm{mL}$, respectively. The results clearly show that resistance to nisin, pediocin 34 and enterocin FH99 did not confer intrinsic resistance to any of the preservatives tested. In no case were the bacteriocin resistant $L$. monocytogenes ATCC 53135 variants examined, resistant to inhibitors than the parental strains.

\section{Discussion}

\section{Kinetics of cell growth inhibition by bacteriocins}

Nisin was observed to be most effective in inhibiting the L. monocytogenes ATCC 53135. It was observed that even when the bacteriocins displayed the most rapid inhibitory activity at $1 \mathrm{~h}$, the survivors resumed growth, reaching the highest cell counts at $24 \mathrm{~h}$. Similar observations were also made by Schillinger et al. (1998), who reported a regrowth of survivors of L. monocytogenes Scott A after exposure to nisin concentrations between 10 and $500 \mathrm{IU}$ $\mathrm{mL}^{-1}$ as well as with those of Song and Richard (1997), who observed that survivors of L. innocua resumed growth after the addition of nisin, pediocin $\mathrm{AcH}$, and enterococcin EFS2 into TSBYE broth. According to Muriana (1996), several studies indicated the immediate decrease of target cells by one to three $\log$ cycles cfu/mL when a bacteriocin was added, with none or little effect on future inoculations. 
Table 5 - Viable cell count (log cfu $\mathrm{mL}^{-1}$ ) of wild type Listeria monocytogenes ATCC 53135 (WT) and its nisin (Nr) resistant, pediocin 34 resistant (Pr) and enterocin FH99 (Er) resistant variant at different time intervals after growth in supplemented with potassium sorbate at concentration of 2.0, 3.0, 4.0, 5.0 and $6.0 \mathrm{mg} / \mathrm{mL}$ (mean \pm standard error, $\mathrm{n}=3$ ).

\begin{tabular}{|c|c|c|c|c|c|c|c|}
\hline \multirow{2}{*}{$\begin{array}{l}\text { Concentration } \\
(\mathrm{mg} / \mathrm{mL})\end{array}$} & \multirow[t]{2}{*}{ Culture } & \multicolumn{6}{|c|}{ Time (h) } \\
\hline & & 1 & 2 & 4 & 6 & 8 & 24 \\
\hline \multirow[t]{4}{*}{ Control } & WT & $7.97 \pm 0.03$ & $7.74 \pm 0.03$ & $8.49 \pm 0.13$ & $7.86 \pm 0.03$ & $8.95 \pm 0.02$ & $8.11 \pm 0.09$ \\
\hline & $\mathrm{Nr}$ & $7.95 \pm 0.04$ & $7.71 \pm 0.03$ & $8.47 \pm 0.01$ & $7.83 \pm 0.09$ & $8.93 \pm 0.08$ & $8.04 \pm 0.03$ \\
\hline & $\operatorname{Pr}$ & $7.99 \pm 0.05$ & $7.72 \pm 0.06$ & $8.86 \pm 0.09$ & $7.83 \pm 0.09$ & $8.94 \pm 0.03$ & $8.00 \pm 0.13$ \\
\hline & $\mathrm{Er}$ & $7.94 \pm 0.09$ & $7.71 \pm 0.03$ & $8.47 \pm 0.01$ & $7.85 \pm 0.02$ & $8.93 \pm 0.09$ & $8.04 \pm 0.03$ \\
\hline \multirow[t]{4}{*}{2.0} & WT & $5.23 \pm 0.04$ & $5.23 \pm 0.09$ & $5.55 \pm 0.03$ & $6.57 \pm 0.04$ & $6.69 \pm 0.06$ & $7.95 \pm 0.04$ \\
\hline & $\mathrm{Nr}$ & $4.59 \pm 0.05$ & $4.83 \pm 0.09$ & $5.44 \pm 0.08$ & $6.53 \pm 0.09$ & $6.32 \pm 0.09$ & $7.77 \pm 0.01$ \\
\hline & $\operatorname{Pr}$ & 5.250 .03 & $5.47 \pm 0.02$ & $5.50 \pm 0.05$ & $6.57 \pm 0.04$ & $6.71 \pm 0.03$ & $7.63 \pm 0.08$ \\
\hline & Er & $4.90 \pm 0.09$ & $5.07 \pm 0.08$ & $4.95 \pm 0.03$ & $5.53 \pm 0.09$ & $5.57 \pm 0.04$ & $6.78 \pm 0.05$ \\
\hline \multirow[t]{4}{*}{3.0} & WT & $4.20 \pm 0.02$ & $4.95 \pm 0.03$ & $5.11 \pm 0.03$ & $5.90 \pm 0.09$ & $6.30 \pm 0.03$ & $6.00 \pm 0.13$ \\
\hline & $\mathrm{Nr}$ & $3.51 \pm 0.04$ & $3.30 \pm 0.03$ & $4.30 \pm 0.03$ & $4.00 \pm 0.12$ & $5.30 \pm 0.11$ & $5.17 \pm 0.01$ \\
\hline & $\operatorname{Pr}$ & $3.30 \pm 0.10$ & $3.38 \pm 0.11$ & $3.47 \pm 0.01$ & $3.50 \pm 0.05$ & $3.30 \pm 0.03$ & $3.90 \pm 0.90$ \\
\hline & Er & $3.56 \pm 0.02$ & $3.41 \pm 0.03$ & $3.67 \pm 0.08$ & $3.75 \pm 0.01$ & $3.77 \pm 0.01$ & $3.81 \pm 0.13$ \\
\hline \multirow[t]{4}{*}{4.0} & WT & $3.44 \pm 0.08$ & $3.60 \pm 0.06$ & $4.00 \pm 0.12$ & $4.20 \pm 0.02$ & 4.250 .03 & $4.47 \pm 0.01$ \\
\hline & $\mathrm{Nr}$ & $2.90 \pm 0.09$ & $2.69 \pm 0.02$ & $3.30 \pm 0.03$ & $3.59 \pm 0.05$ & $3.77 \pm 0.01$ & 3.250 .03 \\
\hline & $\operatorname{Pr}$ & $3.20 \pm 0.02$ & $3.27 \pm 0.04$ & $3.11 \pm 0.02$ & $2.71 \pm 0.03$ & $2.43 \pm 0.04$ & $2.87 \pm 0.06$ \\
\hline & Er & $2.36 \pm 0.08$ & $2.00 \pm 0.09$ & ND & ND & ND & ND \\
\hline \multirow[t]{4}{*}{5.0} & WT & $2.39 \pm 0.04$ & $2.53 \pm 0.09$ & $2.60 \pm 0.06$ & $2.65 \pm 0.03$ & $2.69 \pm 0.07$ & $2.77 \pm 0.01$ \\
\hline & $\mathrm{Nr}$ & $1.90 \pm 0.09$ & $1.95 \pm 0.03$ & $2.30 \pm 0.03$ & $2.39 \pm 0.04$ & $2.30 \pm 0.03$ & $1.95 \pm 0.03$ \\
\hline & $\operatorname{Pr}$ & $2.00 \pm 0.16$ & $2.07 \pm 0.01$ & $2.30 \pm 0.03$ & $2.54 \pm 0.08$ & $2.18 \pm 0.04$ & $2.65 \pm 0.06$ \\
\hline & $\mathrm{Er}$ & 2.250 .03 & $1.74 \pm 0.05$ & ND & ND & ND & ND \\
\hline \multirow[t]{4}{*}{6.0} & WT & $1.87 \pm 0.05$ & $1.60 \pm 0.02$ & $1.47 \pm 0.01$ & $1.54 \pm 0.08$ & $1.60 \pm 0.06$ & $1.47 \pm 0.01$ \\
\hline & $\mathrm{Nr}$ & $1.60 \pm 0.06$ & $1.47 \pm 0.01$ & $1.60 \pm 0.06$ & $1.47 \pm 0.01$ & ND & ND \\
\hline & $\operatorname{Pr}$ & $1.90 \pm 0.09$ & $1.47 \pm 0.01$ & ND & ND & ND & ND \\
\hline & $\mathrm{Er}$ & $1.95 \pm 0.01$ & $1.60 \pm 0.06$ & ND & ND & ND & ND \\
\hline
\end{tabular}

$\mathrm{WT}=$ Wild Type, $\mathrm{Nr}=$ Nisin resistant variant, $\mathrm{Pr}=$ Pediocin 34 resistant variant, Er $=$ Enterocin FH99 resistant variant, ND = Not Detected.

The results of the present study indicate that combinations of different bacteriocins produce a more effective antibacterial effect against L. monocytogenes ATCC 53135 in comparison to the bacteriocins used alone. Similar observations were reported by Hanlin et al. (1993) that a mixture containing more than one bacteriocins would have greater bactericidal effect to a sensitive population, since cells resistant to one bacteriocin might be killed by the other bacteriocin. Moreover synergistic effects were reported when the interactions between pairs of bacteriocins from lactic acid bacteria were tested which are in accordance with the results obtained by Mullet-Powell et al. (1998). The effectiveness of different bacteriocin pairs could be be explained by the fact that the bacteriocins used in this study belonged to different classes, which might vary considerably in the nature and sequence of amino acid residues as also earlier suggested in astudy conducted by Moll et al.
(1999). The synergistic action of combinations of two different bacteriocins with different structures produced by the same strain has also been reported in agar medium by Limonet et al. (2004). Similar results have been reported by Jamuna et al. (2005) who showed that the bacteriocins from $L$. acidophilus and $L$. case $i$ have a better antibacterial activity in combination with Nisin than when used alone against food spoilage and pathogenic organisms in liquid and food systems. Vignolo et al. (2000) also reported that the combined effect of lactocin 705, enterocin CRL35, and nisin against $L$. monocytogenes FBUNT in meat slurry showed no viable counts after incubation for $3 \mathrm{~h}$. Jamuna and Jeevaratnam (2009) have also reported the synergistic effect of Nisin and bacteriocin from Pediococcus acidilactici to be more effective in inhibiting the growth of $L$. monocytogenes and $S$. aureus in sealed pouches of vegetable pulav. 
Table 6 - Viable cell count (log cfu ml ${ }^{-1}$ ) of wild type Listeria monocytogenes ATCC 53135 (WT) and its nisin (Nr) resistant, pediocin 34 resistant (Pr) and enterocin FH99 (Er) resistant variant at different time intervals after growth in supplemented with Sodium Nitrite at concentration of 20, 30, 40, and $50 \mu \mathrm{g} / \mathrm{mL}$ (mean \pm standard error, $\mathrm{n}=3$ ).

\begin{tabular}{|c|c|c|c|c|c|c|c|}
\hline \multirow{2}{*}{$\begin{array}{l}\text { Concentration } \\
(\mu \mathrm{g} / \mathrm{mL})\end{array}$} & \multirow[t]{2}{*}{ Culture } & \multicolumn{6}{|c|}{ Time (h) } \\
\hline & & 1 & 2 & 4 & 6 & 8 & 24 \\
\hline \multirow[t]{4}{*}{ Control } & WT & $7.97 \pm 0.03$ & $7.74 \pm 0.03$ & $8.49 \pm 0.13$ & $7.86 \pm 0.03$ & $8.95 \pm 0.02$ & $8.11 \pm 0.09$ \\
\hline & $\mathrm{Nr}$ & $7.95 \pm 0.04$ & $7.71 \pm 0.03$ & $8.47 \pm 0.01$ & $7.83 \pm 0.09$ & $8.93 \pm 0.08$ & $8.04 \pm 0.03$ \\
\hline & $\operatorname{Pr}$ & $7.99 \pm 0.05$ & $7.72 \pm 0.06$ & $8.86 \pm 0.09$ & $7.83 \pm 0.09$ & $8.94 \pm 0.03$ & $8.00 \pm 0.13$ \\
\hline & $\mathrm{Er}$ & $7.94 \pm 0.09$ & $7.71 \pm 0.03$ & $8.47 \pm 0.01$ & $7.85 \pm 0.02$ & $8.93 \pm 0.09$ & $8.04 \pm 0.03$ \\
\hline \multirow[t]{4}{*}{20} & WT & $6.38 \pm 0.11$ & $6.19 \pm 0.13$ & $6.28 \pm 0.07$ & $6.00 \pm 0.16$ & $7.00 \pm 0.15$ & $6.63 \pm 0.08$ \\
\hline & $\mathrm{Nr}$ & $4.99 \pm 0.06$ & $5.17 \pm 0.09$ & $5.04 \pm 0.09$ & $5.34 \pm 0.03$ & $5.65 \pm 0.13$ & 6.250 .07 \\
\hline & $\operatorname{Pr}$ & $5.39 \pm 0.04$ & 6.250 .03 & $6.30 \pm 0.03$ & $6.15 \pm 0.06$ & $6.01 \pm 0.07$ & $6.09 \pm 0.02$ \\
\hline & $\mathrm{Er}$ & $5.11 \pm 0.03$ & $5.90 \pm 0.09$ & $6.17 \pm 0.01$ & $5.32 \pm 0.09$ & $6.29 \pm 0.03$ & $6.00 \pm 0.06$ \\
\hline \multirow[t]{4}{*}{30} & WT & $4.60 \pm 0.16$ & $4.84 \pm 0.05$ & $4.14 \pm 0.12$ & $4.14 \pm 0.18$ & $4.60 \pm 0.20$ & $4.95 \pm 0.04$ \\
\hline & $\mathrm{Nr}$ & $4.07 \pm 0.09$ & $4.30 \pm 0.10$ & $4.92 \pm 0.09$ & $4.39 \pm 0.04$ & $4.53 \pm 0.10$ & $4.38 \pm 0.11$ \\
\hline & $\operatorname{Pr}$ & $4.00 \pm 0.12$ & $4.41 \pm 0.03$ & $4.87 \pm 0.06$ & $3.90 \pm 0.09$ & $4.44 \pm 0.05$ & $4.39 \pm 0.09$ \\
\hline & $\mathrm{Er}$ & $4.30 \pm 0.03$ & $4.36 \pm 0.08$ & $4.54 \pm 0.18$ & $4.47 \pm 0.03$ & $4.36 \pm 0.06$ & $4.11 \pm 0.08$ \\
\hline \multirow[t]{4}{*}{40} & WT & $3.39 \pm 0.04$ & $3.47 \pm 0.07$ & $3.90 \pm 0.09$ & $4.27 \pm 0.04$ & $4.17 \pm 0.04$ & $3.90 \pm 0.09$ \\
\hline & $\mathrm{Nr}$ & $3.07 \pm 0.08$ & $3.00 \pm 0.06$ & $3.04 \pm 0.04$ & $3.44 \pm 0.07$ & $3.60 \pm 0.06$ & $3.69 \pm 0.07$ \\
\hline & $\operatorname{Pr}$ & $3.04 \pm 0.03$ & $3.08 \pm 0.08$ & $3.55 \pm 0.03$ & $3.47 \pm 0.01$ & $3.65 \pm 0.03$ & $3.69 \pm 0.06$ \\
\hline & $\mathrm{Er}$ & $3.00 \pm 0.10$ & $3.19 \pm 0.05$ & $3.65 \pm 0.07$ & 3.250 .03 & $3.94 \pm 0.08$ & $3.90 \pm 0.09$ \\
\hline \multirow[t]{4}{*}{50} & WT & $3.27 \pm 0.02$ & $3.11 \pm 0.03$ & $3.23 \pm 0.09$ & $3.89 \pm 0.05$ & $3.81 \pm 0.03$ & $3.44 \pm 0.08$ \\
\hline & $\mathrm{Nr}$ & $2.69 \pm 0.07$ & $2.50 \pm 0.05$ & $2.60 \pm 0.06$ & $2.73 \pm 0.04$ & $2.50 \pm 0.05$ & $2.59 \pm 0.06$ \\
\hline & $\operatorname{Pr}$ & $2.90 \pm 0.09$ & $2.97 \pm 0.04$ & $3.00 \pm 0.07$ & $3.07 \pm 0.01$ & $3.27 \pm 0.08$ & $3.01 \pm 0.03$ \\
\hline & $\mathrm{Er}$ & $2.80 \pm 0.08$ & $2.89 \pm 0.05$ & $3.14 \pm 0.05$ & $2.95 \pm 0.03$ & $3.03 \pm 0.06$ & $2.91 \pm 0.08$ \\
\hline
\end{tabular}

$\mathrm{WT}=$ Wild Type, $\mathrm{Nr}=$ Nisin resistant variant, $\mathrm{Pr}=$ Pediocin 34 resistant variant, Er $=$ Enterocin FH99 resistant variant.

\section{Bacteriocin cross resistance}

Several reports suggest that resistance to a bacteriocin may extend to other bacteriocins within the same class or even in other classes. The nisin resistant strain of $L$. monocytogenes has been reported to show cross resistance to the class IIa bacteriocin pediocin PA-1 and the class IV leuconocin S (Crandall and Montville, 1998). $L$. monocytogenes mutants resistant to mesenterocin 52 , curvaticin 13 , and plantaricin were also reported to be crossresistant to the other bacteriocins (Rekhif et al., 1994). In addition, piscicolin 126-resistant mutants of $L$. monocytogenes which emerged in cheese made from milk containing the bacteriocin were also resistant to pediocin P02 (Wan et al., 1997). These reports of cross-resistance indicate that the use of multiple bacteriocins to achieve greater antibacterial efficacy (Hanlin et al., 1993) might not be feasible. Cross-resistance between bacteriocins has also been observed when the sensitivity of Listeria variants to lactocin 705, enterocin CRL35, and nisin was tested. Similar results were obtained by Rekhif et al. (1994) who reported that mutants of L. monocytogenes ATCC 15313 resistant to one of three bacteriocins tested (mesentericin
52, curvaticin 13 , and plantaricin C19), displayed more resistance to the other two, but not to nisin. Insensitivity of a variant to lactocin 705 and enterocin CRL35 while retaining sensitivity to nisin, and vice versa, was associated with the mechanism by which a bacteriocin enters the cell following binding to the cell surface, as well as with the ability to form pores in bacterial membranes.

\section{Cross Resistance of Wild Type and Resistant Strains to Common Food Preservatives}

The application of bacteriocins as part of hurdle technology has received great attention in recent years (Chen and Hoover, 2003; Ross et al., 2003; Deegan et al., 2006). Bacteriocins can be used purposely in combination with selected hurdles in order to increase microbial inactivation. Many reports have reported the ultimate failure of bacteriocin based preservation systems due to the eventual growth of resistant strains (Motlagh et al., 1992; Ming and Daeschel, 1993; Jamuna et al., 2005). Several studies focused in the application of potential synergists of nisin's activity. These included the use of nitrites, low $\mathrm{pH}$, pasteurization, controlled atmosphere, and food ingredients (Rayman et 
al., 1981; Somers and Taylor, 1981; Taylor et al., 1981; Taylor et al., 1985; Motlagh et al., 1992; Rogers and Montville, 1994; Scott and Taylor, 1998a, 1998b) but these reports lacked the data regarding the possible emergence of nisin resistant strains. There are only few reports on the development of intrinsic resistance to other preservation factors. Our results clearly show that resistance to nisin, pediocin 34 and enterocin FH99 did not confer intrinsic resistance to low $\mathrm{pH}$, sodium chloride, potassium sorbate, or sodium nitrite. In contrast, resistant variants were more or equally sensitive than wild-type strain. The resistance mechanism(s) of the nisin, pediocin 34 and enterocin FH99 resistant variants is specific to bacteriocin resistance and do not confer general resistance. Bacteriocins act on sensitive cells by a common mechanism that dissipates the chemical and energy gradient across the cytoplasmic membrane (Okereke and Montville, 1991; Montville and Bruno, 1994). Studies have shown that resistance to nisinconferred cross-resistance to other antimicrobial peptides but did not confer intrinsic resistance to heat (Mazotta and Montville, 1997; Moll et al., 1999) or the other preservative tested in this study, which act on the cell by different mechanisms.

\section{Conclusion}

In conclusion, results presented here indicate that nisin, pediocin 34 and enterocin FH99 in combination have a higher antibacterial action against $L$. monocytogenes ATCC 53135 than when used individually Also the results suggest that resistance to a bacteriocin may extend to other bacteriocins within the same class or even in other classes that might lower the efficacy of bacteriocins when used in combinations. This study also demonstrates that the bacteriocin resistant $L$. monocytogenes variants tested did not become resistant to other preservation factors. The results showed that the nisin, pediocin 34 and enterocin resistant strains of L. monocytogenes ATCC 53135 were generally more preservative-sensitive, therefore, design of hurdle preservation systems containing bacteriocins can improve food safety without being undermined by resistance-related phenomena and also the synergy between different antimicrobial factors might allow the use of lower doses compared to their individual application. Since bacteriocins are considered as potential tools for biopreservation, more study is needed to determine the distribution of bacteriocinresistance phenomena among food borne pathogens.

\section{References}

Biswas SR, Ray P, Johnson MC, Ray B (1991) Influence of growth conditions on the production of a bacteriocin $\mathrm{AcH}$ by Pediococcus acidilactici H. Appl Environ Microbiol 5:1265-1267.

Calvez S, Rince A, Auffray Y, Pre vost H, Djamel D (2007) Identification of new genes associated with intermediate resis- tance of Enterococcus faecalis to divercin V41, a pediocin-like bacteriocin. Microbiol 153:1609-1618.

Chen H, Hoover DG (2003) Bacteriocins and their food applications. Compre Rev Food Science and Food Safety 2:82-100.

Crandall AD, Montville TJ (1998) Nisin resistance in Listeria monocytogenes ATCC 700302 is a complex phenotype. Appl Environ Microbiol 64:231-237.

Dalet K, Cenatiempo Y, Cossart P, Hechard YA (2001) o54-dependent PTS permease of the mannose family is responsible for sensitivity of Listeria monocytogenes to mesentericin Y105. Microbiol 147:3263-3269.

Davies EA, Falahee MB, Adams MR (1996) Involvement of the cell envelope of Listeria monocytogenes in the acquisition of nisin resistance. J Appl Bacteriol 81:139-146.

De Man JC, Rogosa M, Sharpe ME (1960) A medium for the cultivation of lactobacilli. J Appl Bacteriol 2:130-135.

Deegan LH, Cotter PD, Hill C, Ross P (2006) Bacteriocins: biological tools for bio-preservation and shelf-life extension. Int Dairy J 16:1058-1071.

Dykes GA, Hastings JW (1998) Fitness costs associated with class IIa bacteriocin resistance in Listeria monocytogenes B73. Lett Appl Microbiol 26:5-8.

Galvez A, Lopez RL, Abriouel H (2008) Application of Bacteriocins in the Control of Food borne Pathogenic and Spoilage Bacteria. Crit Rev Biotech 28:125-152.

Gravesen A, Ramnath M, Rechinger KB, Andersen N, Jansch L, Hechard Y, Hastings JW, Knøchel S (2002) High-level resistance to class IIa bacteriocins is associated with one general mechanism in Listeria monocytogenes. Microbiol 148:2361-2369.

Gravesen A, Warthoe P, Knochel S, Thirstrup K (2000) Restriction fragment differential display of pediocin-resistant Listeria monocytogenes 412 mutants shows consistent over expression of a putative $\beta$-glucoside-specific PTS system. Microbiol 146:1381-1389.

Gupta H, Malik RK, De S, Kaushik JK (2010) Purification and Characterization of Enterocin FH 99 Produced by a Faecal Isolate Enterococcus faecium $\mathrm{FH}$ 99. Indian J Microbiol 50:145-155.

Hanlin MB, Kalchayanand N, Ray P, Ray B (1993) Bacteriocins of lactic acid bacteria in combination have greater antibacterial activity. J Food Protect 56:252-255.

Harris LJ, Fleming HP, Klaenhammer TR (1992) Developments in nisin research. Food Res Int 25:57-66.

Hechard Y: Pelletier C, Cenatiempo Y, Frere J (2001) Analysis of o54 dependent genes in Enterococcus faecalis: a mannose PTS permease (EII ${ }^{\mathrm{Man}}$ ) is involved in sensitivity to a bacteriocin, mesentericin Y105. Microbiol 147:1575-1580.

Jamuna M, Babusha ST, Jeevaratnam K (2005) Inhibitory efficacy of nisin and bacteriocins from Lactobacillus isolates againast food spoilage and pathogenic organisms in model and food systems. Food Microbiol 22: 449-454.

Jamuna M, Jeevaratnam K (2009). Antibacterial efficacy of nisin and pediocins against food spoilage and pathogenic organisms in broth and a vegetarian food. J Food Sci Technol 46:563-568.

Limonet M, evol-Junelles A, Cailliez-Grimal C, Milliere J (2004) Synergistic mode of action of mesenterocins 52A and 52B produced by Leuconstoc mesenteroides subsp. mesenteroides FR 52. Curr Microbiol 48:204-207. 
Mazzotta AS, Montville TJ (1997) Nisin induces changes in membrane fatty acid composition of Listeria monocytogenes nisin-resistant strains at $10{ }^{\circ} \mathrm{C}$ and $30{ }^{\circ} \mathrm{C}$. J Appl Microbiol 82:32-38.

Ming X, Daeschel MA (1993) Nisin resistance of food borne bacteria and the specific responses of Listeria monocytogenes Scott A. J Food Protect 56:944-948.

Ming X, Daeschel MA (1995) Correlation of cellular phospholipids content with nisin resistance of Listeria monocytogenes Scott A. J Food Prot 58:416-420.

Modi KD (1999) Sensitivity of nisin-resistant Listeria monocytogenes Scott A to heat and preservatives. M.S. Thesis, New Brunswick: Rutgers, the State University of New Jersey, 100 pp. Available from: Rutgers University Library of Science and Medicine. Microbiol 24:53-74.

Moll GN, Konings WN, Driessen AJM (1999) Bacteriocins: mechanism of membrane insertion and pore formation. Antonie Leeuwen 76:185-198.

Montville TJ: Bruno ME (1994) Evidence that dissipation of proton motive force is a common mechanism of action for bacteriocins and other antimicrobial proteins. Int $\mathrm{J}$ Food 24:53-74

Motlagh AM, Holla S, Johnson MC, Ray B, Field RA (1992) Inhibition of Listeria spp. in sterile food system by pediocin $\mathrm{AcH}$, a bacteriocin produced by Pediococcus acidilactici $\mathrm{H}$. J Food Prot 55:337-343.

Mulet-Powell N, Lacoste-Armynot AM, Vinas M, Simeon de Buochberg M (1998) Interactions between pairs of bacteriocins from lactic acid bacteria. J Food Protect 61:12101212.

Muriana PM (1996) Bacteriocins for control of Listeria spp. in food. J Food Prot (Suppl) 54-63.

Naghmouchi K, Drider D, Kheadr E, Lacroix C, Pre vost H, Fliss I (2006) Multiple characterizations of Listeria monocytogenes sensitive and insensitive variants to divergicin M35, a new pediocin like bacteriocin. J Appl Microbiol 100:29-39.

Okereke A, Montville TJ (1991) Bacteriocin-mediated inhibition of Clostridium botulinum spores by lactic acid bacteria at refrigeration and abuse temperatures. Appl Environ Microbiol 57:3423-3428.

Ramnath M, Arous S, Gravesen A, Hastings JW, Hechard Y (2004) Expression of mptC of Listeria monocytogenes induces sensitivity to class IIa bacteriocins in Lactococcus lactis. Microbiol 150:2663-2668.

Ramnath M, Beukes M, Tamura K, Hastings JW (2000) Absence of a putative mannose-specific phosphotransferase system enzyme IIAB component in a leucocin A-resistant strain of Listeria monocytogenes, as shown by two-dimensional sodium dodecyl sulfate-polyacrylamide gel electrophoresis. Appl Environ Microbiol 66:3098-3101.

Rayman MK, Aris B, Hurst A (1981) Nisin: a possible alternative or adjunct to nitrite in the preservation of meats. Appl Environ Microbiol 41:375-380.

Rekhif N, Atrih A, Lefebvre G (1994) Selection and properties of spontaneous mutants of Listeria monocytogenes ATCC
15313 resistant to different bacteriocins produced by lactic acid bacteria strains. Curr Microbiol 28:237-241.

Rogers AM, Montville TJ (1994) Quantification of factors which influence nisin's inhibition of Clostridium botulinum 56A in a model food system. J Food Sci 59:663-669.

Ross AIV, Griffiths MW, Mittal GS, Deeth HC (2003) Combining nonthermal technologies to control foodborne microorganisms. Int J Food Microbiol 89:125-138.

Schillinger U, Chung HS, Keppler K, Holzapfel WH (1998) Use of bacteriocinogenic lactic acid bacteria to inhibit spontaneous nisin-resistant mutants of Listeria monocytogenes Scott A. J Appl Microbiol 85:657-663.

Scott VN, Taylor SL (1981a) Effect of nisin on the outgrowth of Clostridium botulinum spores. J Food Sci 46:117-120.

Scott VN, Taylor SL (1981b) Temperature, $\mathrm{pH}$ and spore load effects on the ability of nisin to prevent the outgrowth of Clostridium botulinum spores. J Food Sci 46:121-126.

Somers EB, Taylor SL (1981) Further studies on the antibotulinal effectiveness of nisin in acidic media. J Food Sci 46:19721973.

Song HJ, Richard J (1997) Antilisterial activity of three bacteriocins used at sub-minimal inhibitory concentrations and cross-resistance of the survivors. Int J Food Microbiol 36:155-161.

Taylor SL, Cann DD, Welch BJ (1990) Antibotulinal properties of nisin in fresh fish packaged in an atmosphere of carbon dioxide. J Food Prot 53:953-957.

Taylor, S.L.; Somers, E.B.; Krueger, L.A. (1985). Antibotulinal effectiveness of nisin-nitrite combinations in culture medium and chicken frankfurter emulsions. J Food Prot 48:234-239.

Uhlman U, Schillinger U, Rupnow JR, Holzapfel WH (1992) Identification and characterization of two bacteriocinproducing strains of Lactococcus lactis isolated from vegetables. Int J Food Microbiol 16:141-151.

Vadyvaloo V, Hastings JW, van der Merwe MJ, Rautenbach M (2002) Membranes of class IIa bacteriocin-resistant Listeria monocytogenes cells contain increased levels of desaturated and short-acylchain phosphatidylglycerols. Appl Environ Microbiol 8:5223-5230.

Verheul A, Russell NJ, Van THR, Rombouts FM, Abee T (1997) Modifications of membrane phospholipid composition in nisin-resistant Listeria monocytogenes Scott A. Appl Environ Microbiol 63:3451-3457.

Vignolo G, Palacios J, Farias ME, Sesma F, Schillinger U, Holzapfel W, Oliver G (2000) Combined effect of bacteriocins on the survival of various Listeria species in broth and meat system. Curr Microbiol 41:410-416.

Wan J, Harmark K, Davidson BE, Hillier AJ, Gordon JB, Wilcock A, Hickey MW, Coventry MJ (1997) Inhibition of Listeria monocytogenes by piscicolin 126 in milk and Camembert cheese manufactured with a thermophilic starter. J Appl Microbiol 82:273- 280.

All the content of the journal, except where otherwise noted, is licensed under a Creative Commons License CC BY-NC. 